\title{
A rigorous examination of the Chandrasekhar theory of stellar collapse
}

\section{Citation}

Lieb, Elliott H., and Horng-Tzer Yau. 1987. "A Rigorous Examination of the Chandrasekhar

Theory of Stellar Collapse." The Astrophysical Journal 323 (December): 140. doi:10.1086/165813.

\section{Published Version}

doi:10.1086/165813

\section{Permanent link}

http://nrs.harvard.edu/urn-3:HUL.InstRepos:32706795

\section{Terms of Use}

This article was downloaded from Harvard University's DASH repository, and is made available under the terms and conditions applicable to Other Posted Material, as set forth at http:// nrs.harvard.edu/urn-3:HUL.InstRepos:dash.current.terms-of-use\#LAA

\section{Share Your Story}

The Harvard community has made this article openly available.

Please share how this access benefits you. Submit a story.

Accessibility 
The Astrophysical Journal, 323:140-144, 1987 December 1

(C) 1987. The American Astronomical Society. All rights reserved. Printed in U.S.A.

\title{
A RIGOROUS EXAMINATION OF THE CHANDRASEKHAR THEORY OF STELLAR COLLAPSE
}

\author{
ElliotT H. LIEB ${ }^{1}$ AND HORNG-TZER YAU ${ }^{1,2}$ \\ Departments of Mathematics and Physics, Princeton University \\ Received 1987 April 20; accepted 1987 May 26
}

\begin{abstract}
Some of the results of a rigorous analysis of the Chandrasekhar semiclassical theory of stellar collapse are presented here. They are of two kinds. The first concerns the Chandrasekhar equation itself; we prove the uniqueness of the solution and also prove that the solution has certain properties not noted before. The second is a derivation of the Chandrasekhar equation from quantum mechanics (in the limit of small gravitational constant) without making a priori assumptions about the smallness of correlation effects. A parallel derivation is made for stars composed of bosons (such as axion stars, if they are ever found to exist); the resulting equation is quite different from the Chandrasekhar equation, for it is of the Hartree type and involves density gradients.
\end{abstract}

Subject headings: stars: collapsed - stars: interiors

\section{INTRODUCTION}

It is a well-known fact that stellar collapse is a complicated phenomenon involving many different factors, e.g., nuclear interaction, thermal radiation, general relativity, etc. However, a fundamental observation that led Chandrasekhar (1931 $a, b$, $c, 1984)$ to obtain his famous mass formula for white dwarfs is the consideration of the equilibrium between the pressure of a degenerate Fermi gas and classical gravitational attraction. In this elementary model, the equilibrium of a nonrotating star is governed by the equation (where $\rho$ is the density and $P$ is the pressure)

$$
-r^{2} \dot{P}(r)=G M(r) \rho(r),
$$

and where $M(r)$ is the mass within radius $r$. The pressure $P(r)$ is determined by assuming a local equation of state $P(\rho)$ which is that of a degenerate Fermi gas. Equation (1), which is what concerns us in this paper, has no general relativistic effects in it, i.e., the Tolman-Oppenheimer-Volkoff equation is not being considered.

It is a remarkable fact that this simple equation predicts collapse and gives the mass limit, $M_{c} \approx 0.7745 m_{n}^{-2} G^{-3 / 2}$, which is at least approximately correct. Although equation (1) was formulated more than half a century ago, and the equation has been analyzed numerically in many cases, especially the polytropic case leading to the Lane-Emden equation, there are several questions which we regard as basic problems concerning equation (1) and which remain to be answered. For example:

1. Given a mass $M$ with $0<M<M_{c}$, is there a solution to equation (1) with the total mass $M(\infty)=4 \pi \int_{0}^{\infty} r^{2} \rho(r) d r=M$ ? If a solution does exist, how many solutions are there?

2. Does every solution to equation (1) describe a stable equilibrium state? More generally, is it an absolute ground state or merely a local minimum (this can be viewed as a question of "global" stability)? In particular, is it true that the spherically symmetric solution (implicit in eq. [1]) has the lowest energy and is therefore the most stable configuration?

3. Is the radius always finite and, if so, what is its dependence

\footnotetext{
${ }^{1}$ Work partially supported by US National Science Foundation grant PHY 85-15288-A01.

${ }^{2}$ Work supported by Alfred Sloan Foundation Dissertation Fellowship.
}

and that of the central density on the stellar mass $M$ ? In particular, is the radius (central density) a decreasing (increasing) function of the mass? Although there exists a simple massradius-central density relation for "polytropic stars" obtained by scaling, it is not obvious that monotonicity holds in the physically interesting, nonpolytropic case of the Fermi gas pressure. It turns out that the radius is finite in the fermion case but not in the boson case.

4. What is the role played by quantum mechanics? More precisely, is it possible to derive equation (1) from a "relativistic" Schrödinger equation? Of course, one expects this quantum-classical correspondence to be exact only in the limit $N \rightarrow \infty, G \rightarrow 0$, but this limit is certainly reasonable physically since $N \approx 10^{57}$ and $m^{2} G \approx 10^{-38}$. Historically, it has been realized since the beginning of quantum mechanics that quantum effects are essential for the stability of stars, e.g., "The black-dwarf material is best likened to a single gigantic molecule in its lowest quantum state" (Fowler 1926). Up to now, however, quantum mechanics entered only through the use of a local equation of state, $P(\rho)$. A first attempt to incorporate the "relativisitic" Schrödinger equation into the study of the stellar collapse problem was made by Lieb and Thirring (1984). The Chandrasekhar value of the critical mass (with the correct exponent, $G^{-3 / 2}$ ) was proved in Lieb-Thirring (1984), but only up to a factor of 4 . Note that a proof of the exact Chandrasekhar value for $M_{c}$ is only one aspect of the "derivation" of equation (1); one also wants to show that equation (1) gives the correct stellar density $\rho(r)$ when $M<M_{c}$. We are aware of the fact that the Schrödinger Hamiltonian we consider here (see eq. [9] below) does not define a Lorentz invariant theory, not to speak of a complete relativistic theory. This would require a formulation of quantum gravity, which is beyond present theoretical technology.

5. What is the semiclassical equation for bosons? In particular, what is the critical mass for "boson stars" (such as axion stars, if they exist)? Since the Pauli exclusion principle does not hold for bosons, the critical mass for bosons is expected to be smaller. It was realized by Thirring (1983) that the correct value for the critical mass should be of order $m G^{-1}$ (instead of the $m G^{-3 / 2}$ appropriate to fermions), thus leading to collapse of objects only the size of a mountain. Other considerations however, may lead to different values (e.g., Stecker and Shafi 
1983). In Ruffini and Bonnazola (1969) and in Lieb and Thirring (1984) it was conjectured that, for bosons, equation (1) should be replaced by a Hartree-type equation. In a sense this would mean there is no semiclassical approximation for bosons (although we shall continue to employ that word) because the Hartree energy involves density gradients $d \rho / d r$, and not just an equation of state. In Lieb-Thirring (1984) the Hartree value of the collapse constant was proved to be correct up to a factor of 2 .

We shall answer the above and related questions rigorously in this paper. Note that equation (1) is a nonlinear differential equation [after using the equation of state $P=P(\rho)$ ], and a direct attack would involve some complicated mathematical machinery (e.g., Ni 1983). Our strategy is to focus instead on a variational problem which has equation (1) as its EulerLangrange equation, as done by Auchmuty and Beals (1971a, $b$ ). Then via a novel interplay between the variational principle and the differential equation we are able to answer questions $1-3$. This is explained in $\S$ II.

As for questions 4 and 5, the reader might wonder why there is any difficulty in deriving the semiclassical equation (1) from quantum mechanics since $N$ is obviously very large $\left(\approx 10^{57}\right)$. The difficulty is an ultraviolet (i.e., short distance) one as we shall now explain. Consider one particle with kinetic energy $T(p)=\left(p^{2}+m^{2}\right)^{1 / 2}-m$ in a gravitational potential $V(x)=-g /|x|$, where $g>0$ is a constant. (We shall henceforth use units in which $c=\hbar=1$.) It is a fact that the Hamiltonian $H=T(p)+V(x)$ is a quantum mechanically unbounded below (i.e., the ground-state energy is $-\infty$ ) if $g>2 / \pi$ (Kato 1966, see remark 5.12, p. 307; Herbst, 1977). For a star, $g \approx G m^{2} \ll 1$, but suppose that, by some fluctuation, $n=3 / \pi\left(G m^{2}\right)^{-1}$ particles come together in a very small region. Since $N \approx\left(G m^{2}\right)^{-3 / 2}, n$ is a very small fraction of $N$, and such a fluctuation is not a priori impossible. Then these $n$ particles will form a trap for other particles, and eventually the whole system will collapse when $N$ is of the order of $\left(G^{2}\right)^{-1}$ instead of $\left(G m^{2}\right)^{-3 / 2}$. [This phenomenon does, in fact occur for bosons, and hence $N_{c}=$ (const) $\left(\mathrm{Gm}^{2}\right)^{-1}$ is correct for bosons.] Although it is intuitively clear that this scenario, in the simple form given above, cannot occur for fermions because of the Pauli principle, it is far from clear that short distance fluctuations do not produce some significant corrections to the semiclassical formula. In fact, they do not do so, but the control of the ultraviolet fluctuations is what complicates the proof of the correspondence. Our results show that there is excellent agreement between the two theories when $N \approx 10^{57}$, e.g., the discrepancy in the ratio of the quantum to the semiclassical critical mass is less than $10^{-4}$.

All the results stated in this paper are rigorously established in a paper of ours (Lieb and Yau 1987). We refer interested readers to that paper for more details and rigorous proofs.

\section{THE SEMICLASSICAL THEORY FOR FERMIONS}

We review briefly how to relate equation (1) to a variational problem (see, e.g., Auchmuty and Beals 1971; Weinberg 1972; Harrison et al. 1965).

Let $\rho(x)$ be the electron number density and

$$
M(r)=\int_{|x| \leq r} d^{3} x \rho(x)
$$

be the (dimensionless) electron number inside the ball of radius $r$. Let $\kappa=G\left(M_{e}+Y_{e} m_{n}\right)^{2}$. Here $Y_{e}$ is the nucleon number per electron, $Y_{e} \approx 2$ for white dwarfs. Let the degenerate electron pressure be given by

$$
P(r)=\frac{8 \pi}{3(2 \pi)^{3}} \int_{0}^{\kappa_{\mathrm{F}}(r)} k^{4}\left(k^{2}+m_{e}^{2}\right)^{-1 / 2} d k
$$

Here $k_{\mathrm{F}}(r)=\left[3 \pi^{2} \rho(r)\right]^{1 / 3}$ is the Fermi momentum. Then the semiclassical equation for gravitational-hydrostatic equilibrium can be written as

$$
-r^{2} \dot{P}(r)=\kappa M(r) \rho(r) .
$$

Note that all mass dimensions have been absorbed into $\kappa$.

Let $a(\rho) \equiv\left(3 \pi^{2} \rho\right)^{1 / 3}$ and

$$
\begin{aligned}
j(\rho) \equiv & \frac{1}{\pi^{2}} \int_{0}^{a(\rho)} p^{2}\left[\left(p^{2}+m_{e}^{2}\right)^{1 / 2}-m_{e}\right] d p \\
= & \left(8 \pi^{2}\right)^{-1}\left\{a\left(2 a^{2}+m_{e}^{2}\right)\left(a^{2}+m_{e}^{2}\right)^{1 / 2}\right. \\
& \left.-m_{e}^{4} \ln \left[a+\left(a^{2}+m_{e}^{2}\right)^{1 / 2}\right]\right\}-\rho m_{e} .
\end{aligned}
$$

Then $j(\rho(x))$ is the semiclassical kinetic energy density for degenerate fermions of number density $\rho$. Let

$$
\mathscr{E}_{\kappa}^{\mathbf{C}}(\rho)=\int d^{3} x j(\rho(x))-\frac{\kappa}{2} \iint d^{3} x d^{3} y \rho(x) \rho(y)|x-y|^{-1}
$$

be the semiclassical energy functional (with $\mathrm{C}$ standing for Chandrasekhar) and let $E_{\kappa}^{\mathrm{C}}(N)$ be the lowest energy of $\mathscr{E}_{\kappa}^{\mathrm{C}}(\rho)$ under the restriction that the total number is $N$, i.e., $\int d^{3} x \rho(x)=N$. By making the variation $\rho \rightarrow \rho+\delta \rho$ with $\int \delta \rho=0$, the Euler-Lagrange equation can be formally calculated to be

$$
\begin{aligned}
& j^{\prime}(\rho(x))=\left\{\left[3 \pi^{2} \rho(x)\right]^{2 / 3}+m_{e}^{2}\right\}^{1 / 2}-m_{e} \\
& \left\{\begin{array}{l}
=\kappa \int d^{3} y \rho(x)|x-y|^{-1}-\mu \text { if } \rho(x)>0 \\
\geq \kappa \int d^{3} y \rho(y)|x-y|^{-1}-\mu \text { if } \rho(x)=0 .
\end{array}\right.
\end{aligned}
$$

Here $\mu$ is a Lagrange multiplier. If $\rho$ is assumed to be radial, then by the usual property of the $r^{-1}$ potential $(|x|=r)$

$$
\int d^{3} y \rho(y)|x-y|^{-1}=r^{-1} M(r)+4 \pi \int_{r}^{\infty} d s s \rho(s) .
$$

Hence equation (3) follows from equation (6) by differentiating equation (6) with respect to $r$.

The semiclassical ground-state energy $E_{\kappa}^{\mathrm{C}}(N)$ is not always finite. It can be shown by numerically solving the Lane-Emden equation of type $4 / 3$ that the critical number $N_{f}(\kappa)$ for the stability of $E_{\kappa}^{\mathrm{C}}(N)$ is independent of $m_{e}$ and can be calculated to be

$$
N_{f}(\kappa)=3.098 \kappa^{-3 / 2} \text {. }
$$

If both sides of equation (8) are multiplied by $\left(m_{e}+Y_{e} m_{n}\right)$, it is clear that the above formula is just the Chandrasekhar mass formula.

We now state our answers to questions $1-3$. We also include some properties of the semiclassical energy $E_{\kappa}^{\mathrm{C}}(N)$, both because of its intrinsic importance and because of its relevance to the study of questions $1-3$. Since equation (5) and equation (6) are translation invariant, any translation of a given solution is still a solution. We shall adopt the convention that all results stated in the following should be understood up to translation, whenever necessary. 
1. Given a total particle number $N$ less than the critical number $N_{f}(\kappa)$, there is exactly one solution to equation (3) with total number $N$. For $N \geq N_{f}(\kappa)$, there is no solution to equation (3) with a total number $N$. Furthermore, as $N$ varies from 0 to $N_{f}(\kappa)$, we obtain all solutions to equation (3), This means, in particular, that there are no spurious solutions to equation (3).

2. The solutions of equation (3) can also be uniquely parametrized by their central densities, i.e., given any central density $\alpha$ (with $0<\alpha<\infty$ ) there is exactly one solution $\rho^{\alpha}$, to equation (3) with central density $\rho^{\alpha}(0)=\alpha$.

3. For each $N<N_{f}(\kappa)$, there is exactly one ground-state $\rho_{N}$ (i.e., a minimizer for eq. [5]) for $E_{\kappa}^{\mathrm{C}}(N)$. It satisfies the EulerLangrange equation (6) for some unique Lagrange multiplier $\mu_{N}$, and $\rho_{N}$ depends only on the radius $r=|x|$. This shows, in particular, that the spherical symmetry assumption can be derived by requiring the solution to be a ground state. For radial functions, equation (6) is equivalent to equation (3), which implies that equation (6) has no spurious radial solutions.

Combining 1, 2, and 3, we have shown that for a star of a given particle number less than the critical number, there is one and only one stable ground state and this state can be obtained by solving equation (3). Conversely, every solution to equation (3) describes the ground state of a star with particle number $N=\int \rho$.

We emphasize that a "stable" state is one that is stable under all perturbations (large or small, radial or not). The stability result we present here is stronger than the usual result where only small radial perturbations are considered (see Shapiro and Teukolsky 1983 for a review and references).

4. $E_{\kappa}^{\mathrm{C}}(N)$ is a strictly concave [i.e., $\left.d^{2} E_{\kappa}^{\mathrm{C}}(N) / d N^{2}<0\right]$, monotone decreasing differentiable function in $\left[0, N_{f}(\kappa)\right]$ with $E_{\kappa}^{\mathrm{C}}(0)=0$ and $E_{\kappa}^{\mathrm{C}}\left[N_{f}(\kappa)\right]=-m_{e} N_{f}(\kappa)$ (see Fig. 1). Let $\mu_{N}$ be the unique Lagrange multiplier associated with $\rho_{N}$. Then $d E_{\kappa}^{\mathrm{C}}(N) / d N=-\mu_{N}$ can be identified as the chemical potential.
5. $\rho_{N}(r)$ is a strictly decreasing function of $r$ and vanishes at some finite radius $R_{N}$. Thus, for every solution to equation (3) there are associated four quantities: the radius $R$, the chemical potential $\mu$, the central density $\alpha$, and the total number $N$. By considering $R, \mu$, and $\alpha$ as functions of $N$ :

(i) $R_{N}$ is a strictly decreasing function of $N ; R_{N}$ tends to zero as $N$ goes to $N_{f}(\kappa)$ and to infinity as $N$ goes to zero.

(ii) $\mu_{N}$ and $\alpha_{N}$ are strictly increasing functions of $N$. Both tend to infinity as $N$ goes to $N_{f}(\kappa)$ and to zero as $N$ goes to zero.

6. For any two stars of different particle numbers, there is exactly one value of $r$ where they have the same density, i.e., $\rho_{1}(r)=\rho_{2}(r)$ (see Fig. 2).

7. For two stars of different particle numbers $N_{1}>N_{2}$, $M_{1}(r)>M_{2}(r)$ for all $r$. Here $M(r)$ is the number of particles inside the sphere of radius $r$ (for stars 1 and 2, respectively). defined in equation (2).

We believe that statements (6) and (7) have not been given before - even heuristically.

\section{THE LARGE $N$ LIMIT OF QUANTUM MECHANICS}

Our starting point for a quantum mechanical treatment of the stellar collapse problem is a "relativistic" Schrödinger Hamiltonian of $N$ gravitating particles of mass $m$ and coupling constant $\kappa$ (in units $\hbar=c=1$ )

$$
H_{\kappa N}=\sum_{i=1}^{N} T_{i}-\kappa \sum_{i<j}^{N}\left|x_{i}-x_{j}\right|^{-1}, \quad T_{i} \equiv\left(p_{i}^{2}+m^{2}\right)^{1 / 2}-m .
$$

Here $p^{2}=-\nabla^{2}$, and the kinetic energy operator $T(p)=\left(p^{2}+m^{2}\right)^{1 / 2}-m$ is most simply understood in terms of the Fourier transform. For white dwarfs, $m=m_{e}$ and $\kappa=$ $G\left(m_{e}+Y_{e} m_{n}\right)^{2}$.

To treat white dwafs properly, one should introduce a Hamiltonian for two kinds of particles, electrons and nuclei, and include Coulomb interactions in addition to the gravitational

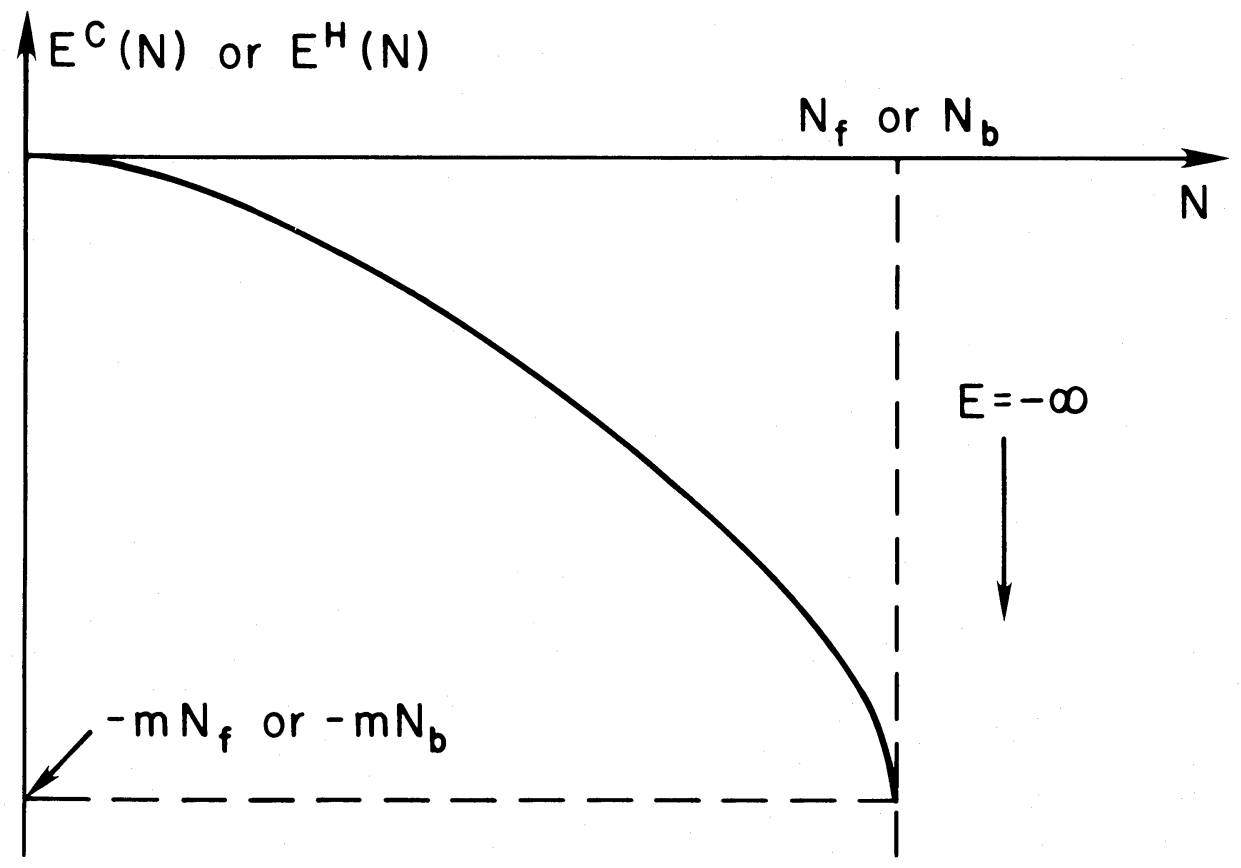

FIG. 1 - The energy of a star as a function of particle number (or mass) for fermions and bosons. Some noteworthy features are (1) the energy is a concave function of $N ;(2)$ the energy is finite at the critical particle number $\left(N_{f}\right.$ or $\left.N_{b}\right)$, but it has an infinite slope there. The figure is schematic. 


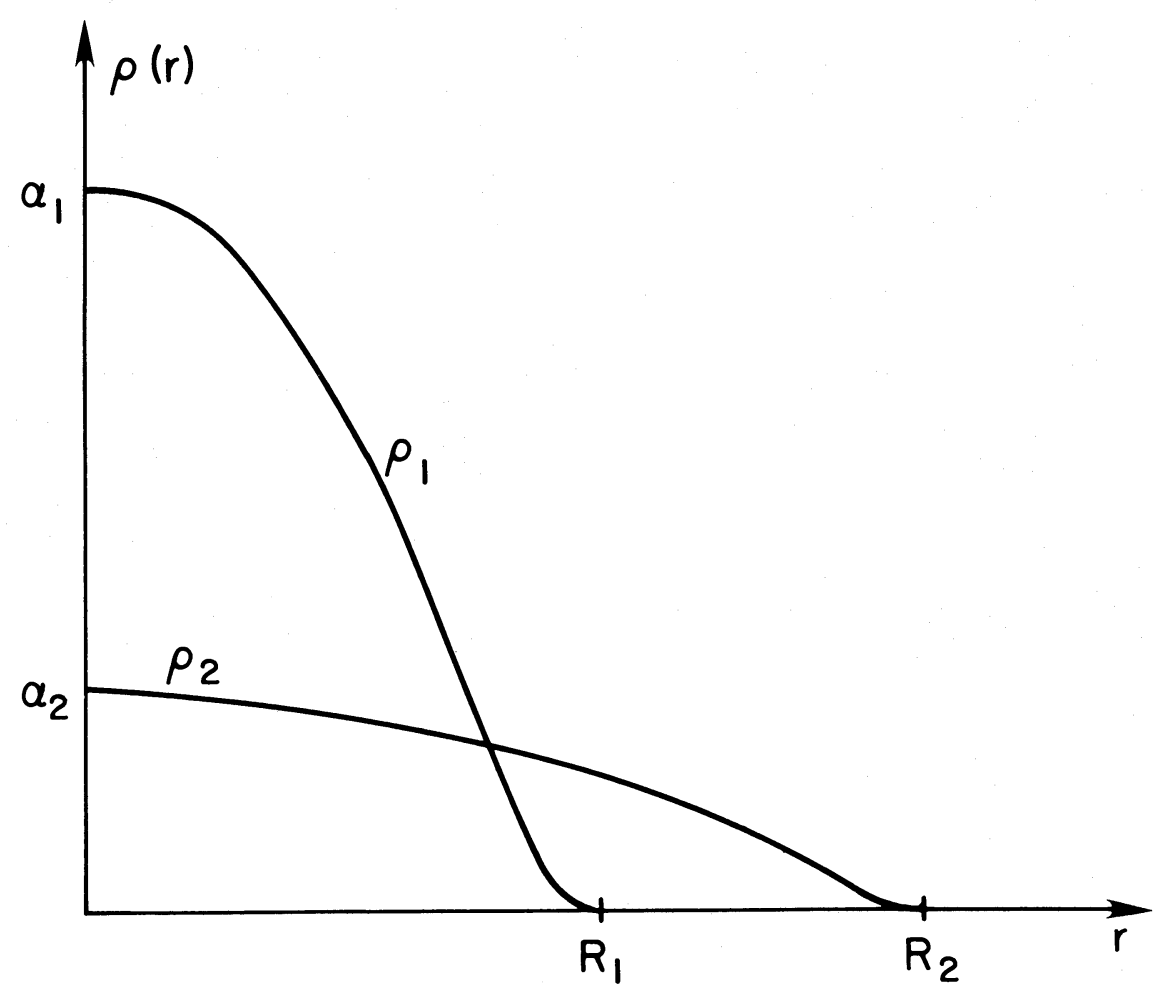

FIG. 2. - Schematics graph of the particle density as a function of radius for two different fermion particle numbers $N_{1}>N_{2}$. Any two such graphs have the property that they intersect at exactly one point.

interaction. That is a much more difficult and unsolved problem. By considering $H_{k N}$ we are making the usual simplifying assumption of local charge neutrality without electrostatic correlation effects and are assuming that the nuclei and electrons are sufficiently correlated that the total gravitational energy is proportional to the electron gravitational energy. $H_{\kappa N}$ is much better as a model for neutron stars (assuming that only neutrons are present in such stars and that nuclear forces can be neglected), but it suffers from the defect that general relativistic effects are ignored. For neutron stars, $m=m_{n}$ and $\kappa=G m_{n}^{2}$ ( $m_{n}=$ neutron mass).

Let $E_{\kappa}^{Q}(N)$ be the quantum ground-state energy of $H_{\kappa N}$ for bosons or fermions as the case may be, and $N_{f}^{Q}(\kappa)\left[\operatorname{resp} . N_{b}^{Q}(\kappa)\right]$ be the critical particle number for the stability of $H_{\kappa N}$ in the fermion [resp. boson] case, i.e., $E_{\kappa}^{Q}(N)=-\infty$ if and only if $N>N^{Q}(\kappa)$. Our results include the following:

1. (fermions):

$$
\lim _{\kappa \rightarrow 0} N_{f}^{Q}(\kappa) / N_{f}(\kappa)=1,
$$

i.e., the Chandrasekhar mass formula is recovered asymptotically. In the physically interesting case (white dwarfs), since $\kappa$ is small $\left(\sim 10^{-38}\right)$, the difference $\left|N_{F}(\kappa)-N_{f}^{Q}(\kappa)\right|$ can be proved to be less than $0.01 \%$ of $N_{f}(\kappa)$.

We also prove that the fractional difference between the quantum ground-state energy and the semiclassical energy is less than $10^{-2}$ if the total particle number $N$ is not too close to the critical value, say $N<0.99 N_{f}(\kappa)$.

2. (fermions): Let $\psi_{N}$ be an approximate ground state of $H_{\kappa N}$ (roughly speaking, an approximate ground state is a state whose energy $\left\langle\psi_{N}\left|H_{\kappa N}\right| \psi_{N}\right\rangle$ is close to the ground-state energy). Then the associated quantum-mechaanical oneparticle density function $\rho_{N}^{Q}$ of $\psi_{N}$ coverages (in an appropriate sense) to a solution of equation (6) as $\kappa \rightarrow 0$.
We remark that the use of "approximate ground state" is physically appropriate and more general than the absolute ground state because when the particle number is huge, it is physical sensible to consider only wave functions $\psi$ which are "near" the ground state.

For a more detailed and precise statement about the convergence of the density function, $\rho_{N}^{Q}$, see Lieb and Yau (1987).

3. (bosons): The Hartree approximation of $H_{\kappa N}$ can be obtained by considering the variational function

$$
\psi\left(x_{1}, \ldots, x_{N}\right) \equiv N^{-N / 2} \prod_{j=1}^{N} \rho\left(x_{j}\right)^{1 / 2},
$$

with $\int d^{3} x \rho(x)=N$. The reesulting Hartree functional is

$$
\begin{aligned}
\mathscr{E}_{\kappa}^{\mathrm{H}}(\rho)=\left\langle\rho^{1 / 2}\right|\left\{\left[p^{2}+m^{2}\right)^{1 / 2}-m\right] & \left|\rho^{1 / 2}\right\rangle-\frac{\kappa}{2} \iint \\
& \times d^{3} x d^{3} y \rho(x) \rho(y)|x-y|^{-1} .
\end{aligned}
$$

The corresponding Euler-Lagrange equation, which replaces equations (3) and (6), is the following Hartree-like equation:

$$
\left[\left(p^{2}+m^{2}\right)^{1 / 2}-m-\kappa U(x)\right] \phi(x)=-\mu \phi(x),
$$

with $\phi(x)=\rho(x)^{1 / 2} \geq 0, p^{2}=-\nabla^{2}$, and $\mu>0$ is the chemical potential. The "potential" $U$ is

$$
U(x)=\int d^{3} y|x-y|^{-1} \rho(y) .
$$

The corresponding ground-state energy and critical number are denoted by $E_{\kappa}^{\mathrm{H}}(N)$ and $N_{b}(\kappa)$. As in the fermion case, we have proved that the quantum value for the ground-state energy and the critical number for stability agree asymptotically with the Hartree values. The actual fractional errors 
for the same $\kappa$ as before $\left(10^{-38}\right)$ are less than $10^{-3}$ and $10^{-4}$ respectively. Indeed, the errors in the boson case are much smaller than those for fermions. We did not calculate the exact Hartree critical value $N_{b}(\kappa)$, but it can be shown that ol $1.27 \kappa^{-1} \leq N_{b}(\kappa) \leq 2.7 \kappa^{-1}$. As in the fermion case, a minimizer $\rho_{N}$, for any given $N$, is necessarily spherically symmetric and radially decreasing.

It is difficult to interpret equation (10) simply as an equation for gravitational-hydrostatic equilibrium. Nevertheless, it is correct. Equation (10) is a kind of nonlinear Schrödinger equation, and, like any Schrödinger equation, its solution will not vanish at some finite $R$. A bosonic star will have a tail extending to infinity.

We are grateful to C. King, M. Loss, J. Peebles, and W. Thirring for helpful conversations.
Auchmuty, J., and Beals, R. 1971a, Arch. Rat. Mech. Anal. 43, 255. $1971 b, A p . J$. (Letters) 165, L79.

Chandrasekhar, S. 1931a, Phil. Mag., 11, 592. 1931b,Ap.J., 74,81.

1931c, M.N.R.A.S., 91, 456.

1931c,M.N.R.A.S., 91, 456.

Fowler, R. H. 1926, M.N.R.A.S.,87, 114

Harrison, B. K., Thorne, K. S., Wakano, M., and Wheeler, J. A. 1965, Gravitational Theory and Gravitational Collapse (Chicago: University of Chicago Press).

Herbst, I. 1977, Comm. Math. Phys., 53, 285; 55, 316.

\section{REFERENCES}

Kato, T. 1966, Perturbation Theory For Linear Operators (Berlin: Springer). Lieb, E., and Thirring, W. 1984, Ann. Phys., 155, 494.

Lieb, E., and Yau, H-T. 1987, Comm. Math. Phys., 112, 147.

Ni, W. M. 1983, J. Diff. Eq., 5, 289.

Ruffini, R. and Bonazzola, S. 1969, Phys. Rev., 187, 1767.

Shapiro, S., and Teukolsky, S. 1983, Black Holes, White Dwarfs and Neutron Stars (New York: Wiley-Interscience).

Stecker, F. and Shafi, Q. 1983, Phys. Rev. Letters, 50, 925.

Thirring, W. 1983, Phys. Letters B, 127, 27.

Weinberg, S. 1972, Gravitation and Cosmology (New York: Wiley).

Elliott H. Lieb AND Horng-Tzer YAU: Department of Physics, Joseph Henry Laboratories, Jadwin Hall, P.O. Box 708, Princeton, NJ 08544 